\title{
Endometriosis Involving Colon and Rectum: A Literature Review and Laparoscopic Management
}

\author{
Abdallah Elsabagh ${ }^{1 *}$ and Yik Hong $\mathrm{Ho}^{2}$ \\ ${ }^{1}$ Department of General Surgery, Fiona Stanley hospital, Western Australia, Australia \\ ${ }^{2}$ Discipline of surgery, James Cook University, Townsville, Queensland, Australia \\ *Corresponding author: Abdallah Elsabagh, Department of General Surgery, Fiona Stanley Hospital, Australia
}

\begin{abstract}
Introduction: Endometriosis is characterized by the presence of functional endometrial tissue consisting of glands and/ or stroma located outside the uterus [1], although implanted ectopically, this tissue presents histopathological and physiological responses that are similar to the responses of the endometrium [2].

Clinical Features: Endometriosis usually becomes apparent in the reproductive years when the lesions are stimulated by ovarian hormones. Forty percent of the patient's present symptoms in a cyclic manner, which are usually related with menses Pelvic pain, infertility and dyspareunia are the characteristic symptoms of the disease, but the clinical presentation is often non-specific [1].

Diagnosis and Investigations: A precise diagnosis about the presence, location and extent of rectosigmoid endometriosis is required during the preoperative workup because this information is necessary in the discussion with both the colorectal surgeon and the patient. Furthermore, almost all patients with intestinal endometriosis have lesions in multiple pelvic locations and it is difficult to know what symptoms are caused by the intestinal disease versus the pelvic disease.

Treatment: Treatment must be individualized, taking the clinical problem in its entirety into account, including the impact of the disease and the effect of its treatment on quality of life. Pain symptoms may persist despite seemingly adequate medical and/ or surgical treatment of the disease. In such circumstances, a multi-disciplinary approach involving a pain clinic and counselling should be considered early in the treatment plan.
\end{abstract}

\section{Introduction}

Endometriosis is characterized by the presence of functional endometrial tissue consisting of glands and/ or stroma located outside the uterus [1], although implanted ectopically, this tissue presents histopathological and physiological responses that are similar to the responses of the endometrium [2].

\section{Prevalence and Epidemiology}

The reported prevalence of endometriosis is $1 \%-20 \%$ in asymptomatic women, $10 \%-25 \%$ in infertile patients and $60 \%$ $70 \%$ in women with chronic pelvic pain [1]. Endometriosis is a common benign disease among women of reproductive age and affects the intestinal tract in $15 \%-37 \%$ of all patients with pelvic endometriosis [3]. Multiple births and extended intervals of lactation decrease the risk of being diagnosed with endometriosis, whereas nulliparity, early menarche, frequent menses, and prolonged menses increase the risk [4]. Endometriosis also appears to be associated with a taller, thinner body habitus and lower body mass index [5]. The prevalence appears to be lower in blacks and Asians than in Caucasians [6]. Growth and maintenance of endometriotic implants are dependent upon the presence of ovarian steroids. As a result, endometriosis occurs during the active reproductive period: women aged 25 to 35 years [6]. Other factors that appear to play important roles in determining if a woman will develop the clinical condition include [7]:
a) Reproductive lifestyle, especially a delay in childbearing
b) Poorly understood immunological factors
c) Some environmental factors, probably including exposure to a range of environmental toxins 
d) Reproductive tract occlusion, such as an imperforate hymen.

\section{Pathogenesis}

Endometriosis is a common disease of unknown etiology. Many theories have been proposed to explain this condition: retrograde menstruation theory, metaplastic, transformation, the migration of cells through the lymphatic system or via hematogenous spread, Iatrogenic during CS. However, other factors, immunological, genetic and familial, could be involved in the pathogenesis of this disease [1].

\section{Sampson's Theory of Retrograde Menstruation}

The implantation theory proposes that endometrial tissue passes through the fallopian tubes during menstruation, then attaches and proliferates at ectopic sites in the peritoneal cavity. Recent studies using laparoscopy have demonstrated that retrograde menstruation is a nearly universal phenomenon in women with patent fallopian tubes. Classic studies performed in the 1950s demonstrated viability of sloughed endometrial cells and the capacity to implant at ectopic sites. Patients with mullerian anomalies and obstructed menstrual flow through the vagina may have an increased risk of endometriosis. The anatomic distribution of endometriosis also provides evidence for Sampson's theory [8].

\section{Coelomic Metaplasia Theory}

The theory of coelomic metaplasia proposes that endometriosis may develop from metaplasia of cells lining the pelvic peritoneum. Iwanoff and Meyer are recognized as originators of this theory. A prerequisite of the coelomic metaplasia theory is that mesothelial cells lining the ovary and pelvic peritoneum contain cells capable of differentiating into endometrium. An attractive component of the coelomic metaplasia theory is that it can account for the occurrence of endometriosis anywhere mesothelium is found. This includes reports of endometriosis occurring in the pleural cavity. Pleural endometriosis could result from local metaplasia of pleural mesothelium. On the other hand, it could also result from transdiaphragmatic passage of peritoneal fragments of endometrium as well as vascular metastasis of endometrium. Coelomic metaplasia is thought to account for the rare occurrences of endometriosis reported in males. In these reports of endometriosis, the men were all undergoing estrogen therapy. Although coelomic metaplasia was a possibility, estrogen stimulation of mullerian rests could not be excluded. Likewise, the occurrence of endometrial carcinoma in males is thought to possibly arise from mullerian remnants. Still, further support for the coelomic metaplasia theory may be found in the study of benign and malignant epithelial ovarian tumors. Both are considered to be derivatives of germinal epithelium. The presence of ovarian surface endometriosis could be accounted for by this type of transformation [8].

\section{Induction Theory}

The induction theory is an extension of the coelomic metaplasia theory. This theory proposes that menstrual endometrium produces substances that induce peritoneal tissues to form endometriotic lesions [8].

\section{Embryonic Rests Theory}

Von Recklinghausen and Russell are credited with the theory that endometriosis results from embryonic cell rests. These embryonic rests, when stimulated, could differentiate into functioning endometrium. As described above, rare cases of endometriosis have been reported in men. Transformation of embryonic rests is a plausible explanation for this phenomenon [8].

\section{Lymphatic and Vascular Metastasis Theories}

The lymphatic metastasis theory of endometriosis is often referred to as Hal ban's theory. He reported that endometriosis could arise in the retroperitoneum and in sites not directly opposed to peritoneum. Sampson had also suggested that endometriosis could result from lymphatic and hematogenous dissemination of endometrial cells. An extensive communication of lymphatics has been demonstrated between the uterus, ovaries, tubes, pelvic and vaginal lymph nodes, kidney, and umbilicus. Metastasis of endometrial cells via the lymphatic system to these areas is therefore anatomically possible. These findings are consistent with a literature review showing a $6.7 \%$ incidence of lymph node endometriosis in 178 autopsy cases. Lymphatic and vascular metastasis of endometrium has been offered as an explanation for rare cases of endometriosis occurring in locations remote from the peritoneal cavity. In addition to pleural tissue, endometriosis has been reported in pulmonary parenchyma. Vascular or lymphatic metastasis may also explain cases of endometriosis that have been reported in bone, biceps muscle, peripheral nerves, and the brain [8].

\section{Composite Theory}

Javert proposed a composite theory of the histogenesis of endometriosis which combines the implantation, vascular/ lymphatic metastasis, as well as a theory of direct extension of endometrial tissue through the myometrium. Along similar lines, Nisolle and Donnez have recently argued that the histogenesis of endometriosis depends on the location and 'type' of the endometriotic implant. For example, peritoneal endometriosis can be explained by the implantation theory. Ovarian endometriomas could be the result of coelomic metaplasia of invaginated ovarian epithelial inclusions. Rectovaginal endometriosis, which often resembles adenomyosis, could result from metaplasia of Mullerian remnants located in the rectovaginal septum. These composite theories are attractive in that they recognize a multifaceted mechanism of histogenesis. It seems logical that a disease with such variable manifestations may originate via several mechanisms [8].

\section{Altered Immunity}

Alterations in immunologic response to retrograde menstruation have been implicated in the genesis and maintenance of the endometriotic lesion. This defective immunosurveillance may lead to decreased clearance of menstrual debris from the peritoneal cavity and may allow for attachment of ectopic endometrium to peritoneal surfaces. An abnormal immune response could also promote the persistence and growth of ectopic endometrial tissue [8]. 


\section{The "Neurologic Hypothesis"}

It is a new concept in the pathogenesis of endometriosis: There is a close histological relationship between endometriotic lesions of the large bowel and the nerves of the large bowel wall. Endometriotic lesions seem to infiltrate the large bowel wall preferentially along the nerves, even at distance from the palpated lesion, while the mucosa is rarely and only focally involved [9].

\section{Pathology and Sites of Involvement}

\section{Sites}

Endometriosis can be divided into intra- and extraperitoneal sites. In decreasing order of frequency, the intra-peritoneal locations are ovaries (30\%), uterosacral and large ligaments (18\%-24\%), fallopian tubes (20\%), pelvic peritoneum, pouch of Douglas, and gastrointestinal (GI) tract. Extra-peritoneal locations include cervical portio $(0.5 \%)$, vagina and rectovaginal septum, round ligament and inguinal hernia sac (0.3\%-0.6\%), navel (1\%), abdominal scars after gynaecological surgery $(1.5 \%)$ and caesarian section $(0.5 \%)$. Endometriosis rarely affects extraabdominal organs such as the lungs, urinary system, skin and the central nervous system [1]. Endometriosis affects the intestinal tract in $15 \%$ to $37 \%$ of patients with pelvic endometriosis [10], involvement have been reported from the small bowel to the anal canal, but more frequently the disease involves the rectum and the sigmoid colon (74\%), followed by the rectovaginal septumn (12\%), cecum (2\%), and appendix (3\%) . When the ileum is involved, the most common tract is the distal part. A full-thickness involvement of the colonic wall is infrequent since the mucosa is usually spared. One of the classic locations is the anterior rectal wall in the region of the pouch of Douglas. This can be single nodule or can simulate a cancer. Because of the invasive appearance, the disease can be mistaken for cancer [11].

\section{Gross and Microscopic Pathology of Bowel Endometriosis}

The appearance and size of the implants are quite variable. Areas of endometriosis appear as raised flame-like patches, whitish opacifications, yellow-brown discoloration, translucent blebs, or reddish or reddish-blue irregularly shaped islands. The peritoneal surface may be scarred or puckered.

\section{The Microscopic Appearance}

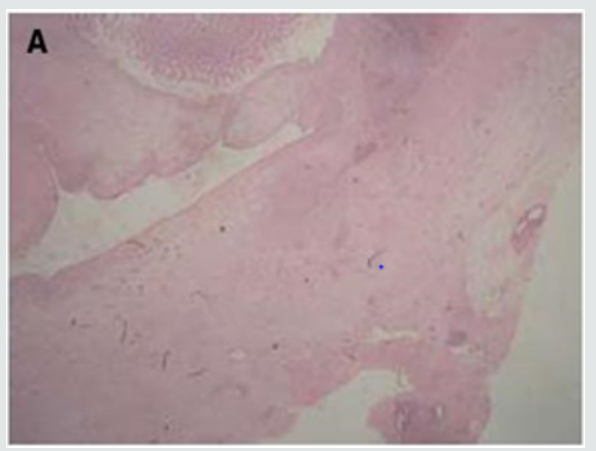

Figure 1(a): Low-power image of the colonic wall, with a few endometrial glands and stroma embedded in the muscular layer.
Of endometriotic tissue is similar to that of endometrium in the uterine cavity; the two major components of both are endometrial glands and stroma. Unlike endometrium, however, endometriotic implants often contain fibrous tissue, blood, and cysts (Figure 1(a) $\& 1(b))$.

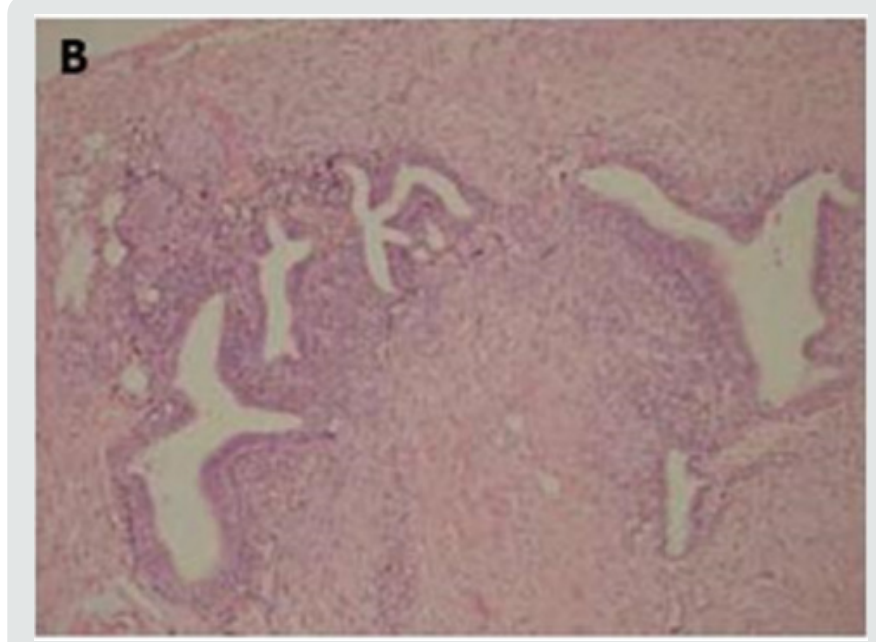

Figure 1(b): High-power view of the colonic wall, with endometrial glands and stroma embedded in the smooth muscle of the colon [12].

\section{Link to Cancer}

Endometriosis is considered a benign disorder; however, it shares some of the characteristics of malignancy, such as abnormal morphology, deregulated cell growth, cellular invasion, and neoangiogenesis. The glandular epithelium occasionally displays DNA aneuploidy. In vitro evidence suggests that endometriosis may have a monoclonal origin. In addition to being monoclonal, endometriotic deposits showed loss of heterozygosity in $28 \%$ of lesions. In 2002, Nezhat et al. with immunohistochemistry, found that alterations in bcl-2 and p53 may be associated with the malignant transformation of endometriotic cysts [12]. The development of a malignancy is a relatively common complication of endometriosis. In fact, several publications have reported malignant neoplasms arising from endometriosis. Most of these publications are case reports or refer to a small series of patients presenting either ovarian carcinomas with associated endometriosis or invasive endometrioid adenocarcinomas involving adjacent pelvic structures. Malignant transformation of extraovarian endometriosis, including the intestinal tract, however, has not been reported as frequently. The largest reported series of neoplastic changes in gastrointestinal endometriosis includes 17 cases [10] (Figure 2(a-d)). Some studies suggest that the development of malignancies may occur in up to $5.5 \%$ of female patients with endometriosis. Only $21.3 \%$ of the cases arise from extragonadal pelvic sites, and endometriosis-associated intestinal tumors are even rarer. Malignant transformation of primary gastrointestinal endometriosis without pelvic involvement is uncommon, and its real incidence is unknown. It can mimic a primary gastrointestinal neoplasm. Most of these neoplasms are carcinomas, but sarcomas and müllerian adenosarcomas 
have also been described. Petersen et al, in a large review of the previously published endometrioid adenocarcinomas arising in colorectal endometriosis, report less than 50 cases of neoplastic transformation, 22 of which were adenocarcinomas. The others included sarcomas and mixed müllerian tumors. The progression to invasive cancer has been related with hyperestrogenism, either of endogenous or of exogenous origin. A possible genetic background favoring the onset of cancer has been reported in some patients without hyperestrogenism and with a family history of cancer. The anatomic distribution and frequency of these cancers parallel the occurrence of which benign endometriosis is found at various sites. In order to classify a malignancy as arising from endometriosis, strict histopathologic criteria need to be fulfilled. Sampson first proposed these criteria in the year 1925. He suggested that the following should be fulfilled:

a) the presence of both malignant and benign endometrial tissue in the same organ.

b) the demonstration of cancer arising in the tissue and not invading it from elsewhere.

c) the finding of tissue resembling endometrial stroma surrounding characteristic glands.

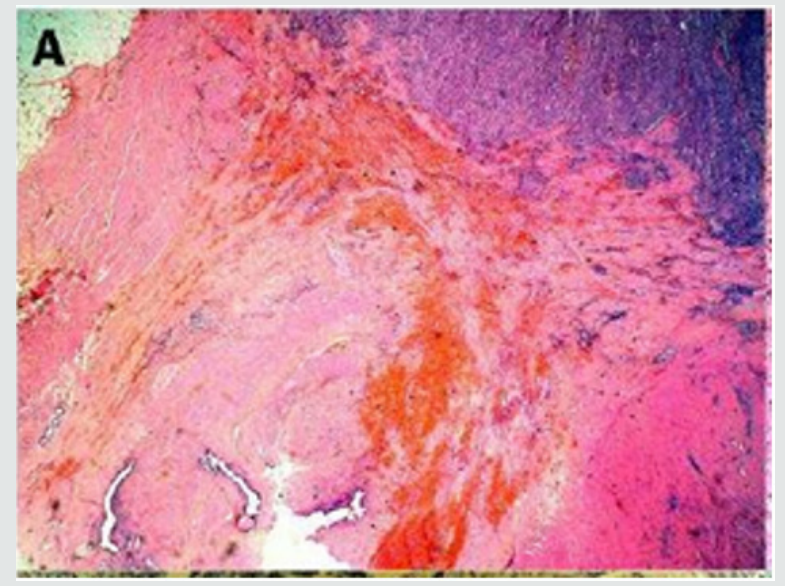

Figure 2(a): Rectal endometriod adenocarcinoma with adjacent focus of endometriosis (hematoxylin-eosin, 20x).

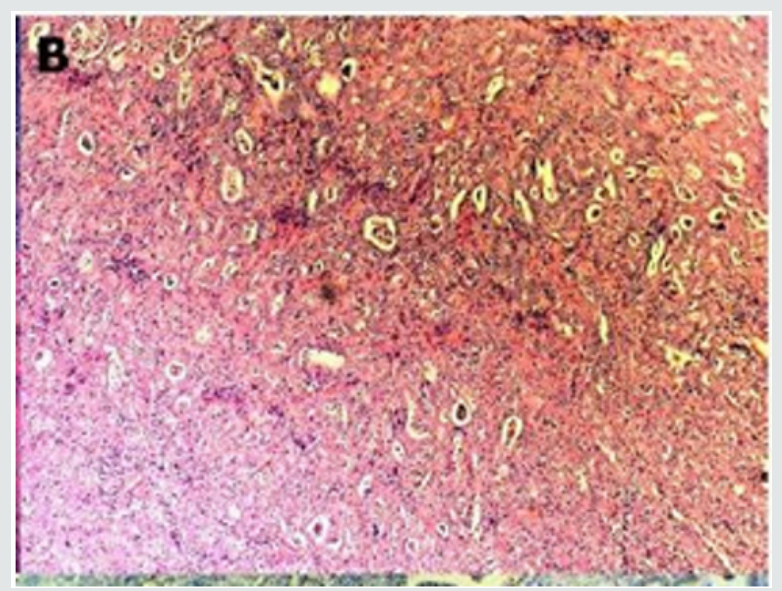

Figure 2(b): Rectal endometriod adenocarcinoma endometriosis (hematoxylin-eosin, 100x).

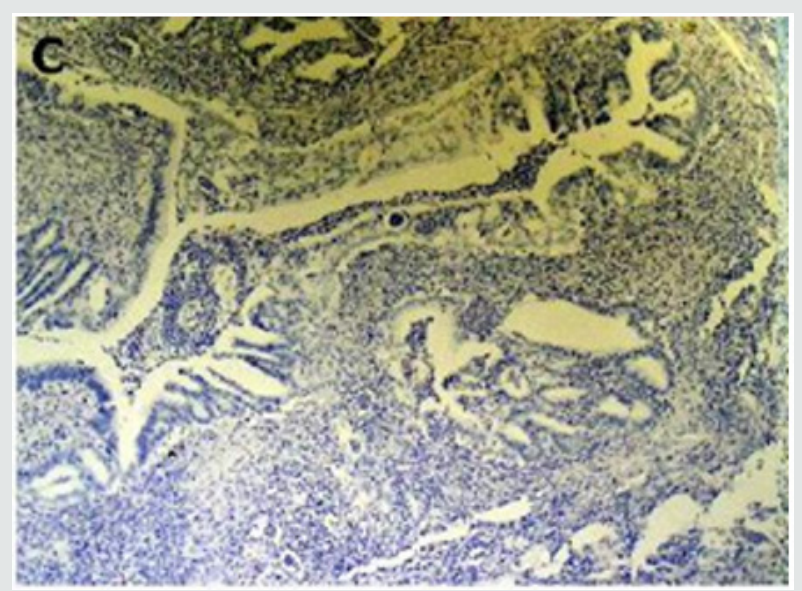

Figure 2(c): Cytokeratin 20 immunostaining negative (100x).

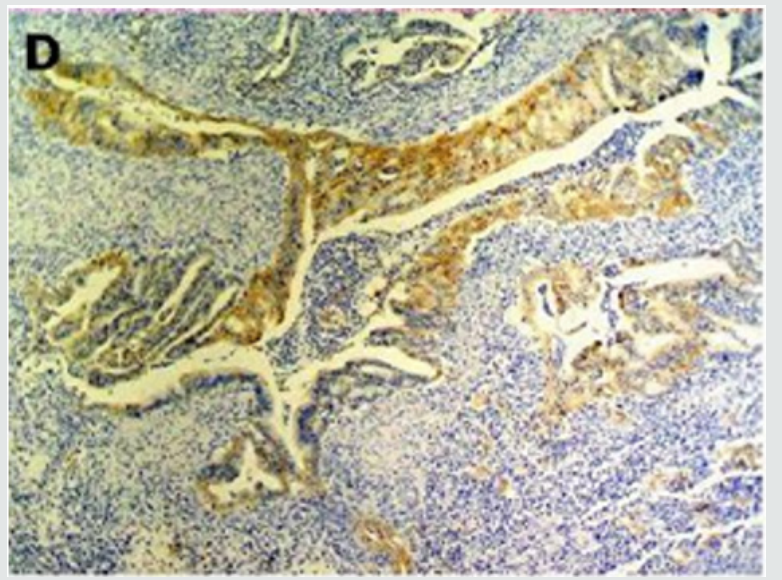

Figure 2(d): Cytokeratin 7 immunostaining positive (100x). [10]

Years later, Scott suggested an additional qualification to complete Sampson's criteria: the demonstration of microscopic benign endometriosis contiguous with the malignant tissue [10]. Endometriosis and its possible malignant changes should be taken into account in the differential diagnosis of intestinal masses in females. Also, clinical suspicion for malignancy should be aroused in patients with abdominal pain or rectal bleeding and a previous history of quiescent endometriosis. Recognition of these lesions is important because of the different management required by primary gastrointestinal neoplasms and by those arising from endometriosis. These differences may have significant clinical implications [10].

\section{Clinical Features}

Endometriosis usually becomes apparent in the reproductive years when the lesions are stimulated by ovarian hormones. Forty percent of the patient's present symptoms in a cyclic manner, which are usually related with menses Pelvic pain, infertility and dyspareunia are the characteristic symptoms of the disease, but the clinical presentation is often non-specific [1]. Symptoms are initially cyclical but may become permanent when the lesions progress. It is difficult to establish a preoperative diagnosis of GI endometriosis, 
because GI tract symptoms can mimic a wide spectrum of diseases, including irritable bowel syndrome, infectious diseases, ischemic enteritis/colitis, inflammatory bowel disease and neoplasm. GI endometriosis patients present with relapsing bouts of abdominal pain, abdominal distention, tenesmus [1], constipation and diarrhoea. Rectal bleeding and pain during defecation may also occur. Endometriosis infiltrating the muscularis propria may lead to localized fibrosis in the bowel wall, strictures, and small or large bowel obstruction. The true incidence of endometriosis causing bowel obstruction is unknown, although complete obstruction of Table 1. the bowel lumen occurs in less than $1 \%$ of cases. Endometriosis of the distal ileum is an infrequent cause of intestinal obstruction, ranging from $7 \%$ to $23 \%$ of all cases with intestinal involvement. The incidence of intestinal resection for bowel obstruction is $0.7 \%$ among patients undergone surgical treatment for abdominopelvic endometriosis [1]. Rectal bleeding may be caused by mucosal injury during the passage of stools through a stenosed colon with the intramural endometriotic tissue increased at the time of menses if it occurs. Colonic mucosa heals rapidly, and no signs are detectable at endoscopy [1] (Table 1).

\section{Summary [1]}

1. the clinical presentation is often non-specific

2. Symptoms are initially cyclical but may become permanent when the lesions progress

3. Pelvic pain, infertility and dyspareunia are the characteristic symptoms of the disease

4. It is difficult to establish a preoperative diagnosis of GI endometriosis

5. GI endometriosis patients present with relapsing bouts of abdominal pain, abdominal distention, tenesmus, constipation and diarrhoea. Rectal bleeding and pain during defecation may also occur.

6. Endometriosis infiltrating the muscularis propria may lead to localized fibrosis in the bowel wall, strictures, and small or large bowel obstruction

\section{Differential diagnosis [1]}

a) irritable bowel syndrome,

b) infectious diseases,

c) ischemic enteritis/colitis,

d) inflammatory bowel disease

e) neoplasm

f) Other causes of intestinal obstruction (Acute/chronic, small/large bowel)

\section{Diagnosis and Investigations}

A precise diagnosis about the presence, location and extent of rectosigmoid endometriosis is required during the preoperative workup because this information is necessary in the discussion with both the colorectal surgeon and the patient. Furthermore, almost all patients with intestinal endometriosis have lesions in multiple pelvic locations and it is difficult to know what symptoms are caused by the intestinal disease versus the pelvic disease. In particular, in the case of sigmoid endometriosis, the lesion cannot be suspected at clinical examination, which is why sigmoid endometriosis is often diagnosed only during surgery. Although several radiological techniques have been proposed for the diagnosis of bowel endometriosis, data are inconclusive, and no gold standard is currently available [13].

\section{Colonoscopy}

Although endoscopic diagnosis of colonic endometriosis has been reported, the mucosa is usually normal or shows minimal mucosal abnormalities, friability, extrinsic process or fibroses stenoses [1]. Endoscopic biopsies usually yield insufficient tissue for a definitive pathologic diagnosis as endometriosis involves the deep layers of the bowel wall [14]. Endometriosis can induce mucosal changes without any specific pattern, which mimic findings of other diseases such as inflammatory bowel disease, ischemic colitis or neoplasm [1]. Colonoscopy is helpful to rule out colorectal malignancy [11].

\section{Double Contrast Barium Enema}

Radiologically, lesions of endometriosis are either of constricting and polypoid type or both. On barium studies, radiographic findings caused by implants in the ileum are similar to those in the colon. Rectosigmoid or cecal endometriosis on double contrast barium enema studies is seen as an extrinsic mass with speculation and tethering of folds [1]. Shortening or flattening of the bowel wall, crenulation of the mucosa, or a combination of these factors [15], Double-contrast barium enema may be effective in determining the precise location of the endometriotic nodules, but it cannot clearly demonstrate the depth of parietal involvement. Furthermore, the experience of the radiologist in the diagnosis of bowel endometriosis remains a critical limit of this technique [13] (Figures $3 \& 4(a \& b)$ ). 

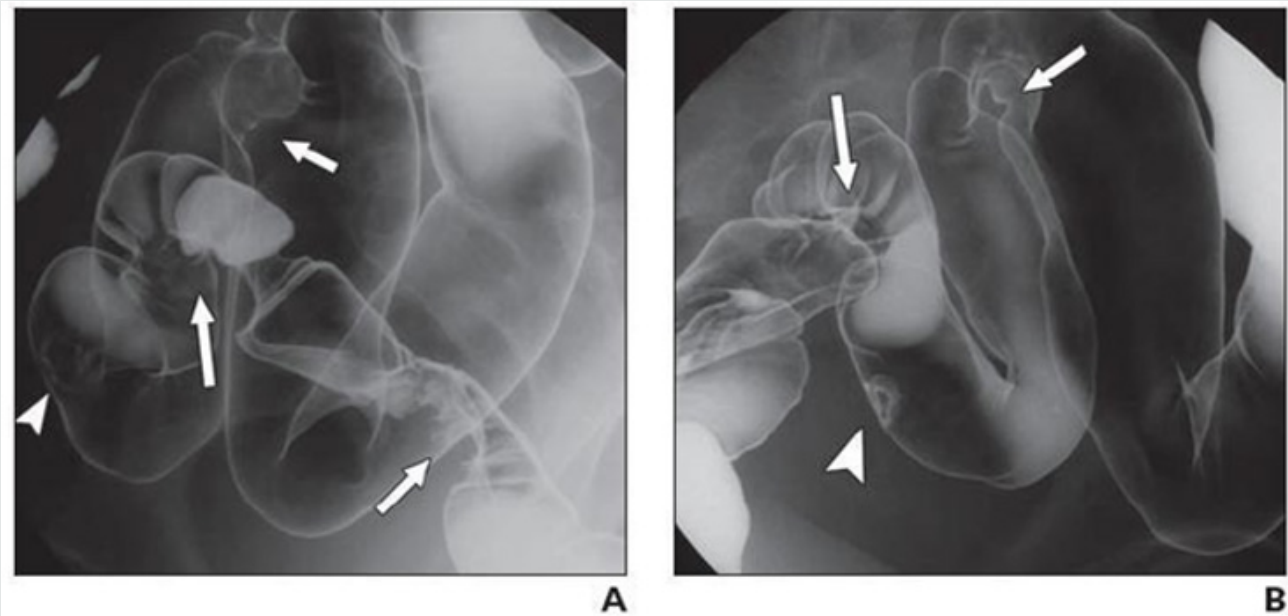

Figure 3: Thirty-four years old woman with suspected intestinal implants of endometriosis. A and B, Lateral A and oblique B spot images show three endometriotic lesions exhibiting extrinsic mass effect with crenulation of contour and speculation that are direct signs of infiltration of bowel wall (arrows). Small polypoid lesion (arrowhead) is benign tubular adenoma confirmed at surgery [15].

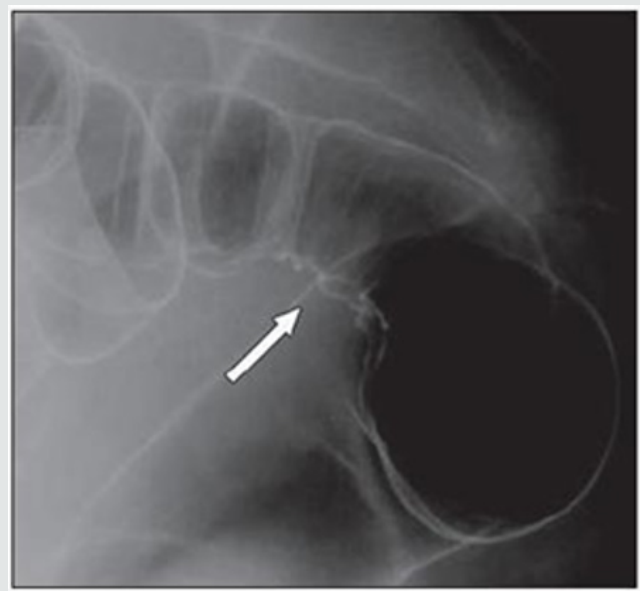

Figure 4(a): Twenty-eight years old woman with suspected intestinal implants of endometriosis and finding of rectal localization of intestinal endometriosis. DCBE image shows extrinsic mass effect and speculation (arrow) of rectal wall that appears infiltrated [15].

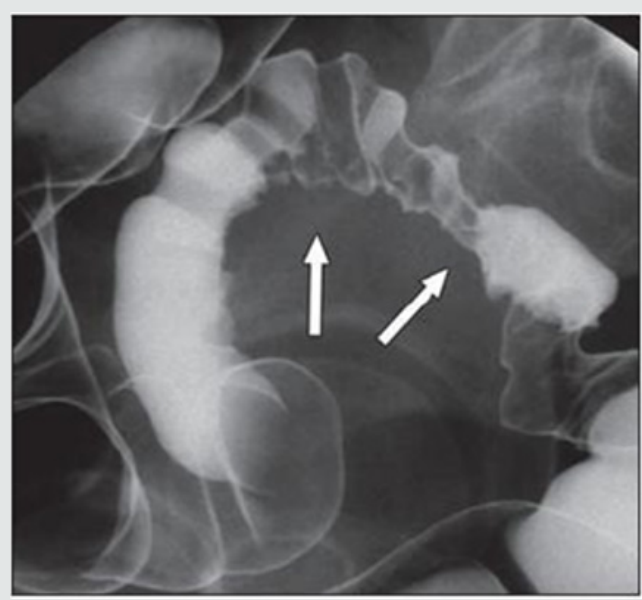

Figure 4(b): Twenty-three years old woman with suspected intestinal implants of endometriosis. DCBE examination showing pathologic pelvic process involving bowel serosa at rectosigmoid junction. Finding of extrinsic mass effect and speculation (arrows) owing to poor wall distention after air insufflation suggesting wall infiltration [15]. 


\section{Transvaginal Us}

Transvaginal ultrasonography can be useful not only in the first-line exploration of the pelvic cavity, but also in diagnosing rectosigmoid endometriosis. However, relevant limitations of transvaginal ultrasonography consist in the impossibility of determining the exact distance of rectal lesions from the anal margin and of evaluating precisely the depth of rectal wall involvement. In addition, locations above the rectosigmoid junction might be beyond the field of view of a transvaginal approach and limited by the presence of air for a transabdominal approach [15]. Transvaginal us combines with rectal water contrast is more accurate than TVS in diagnosing rectal infiltration reaching at least the muscularis propria in women with rectovaginal endometriosis. However, this exam cannot determine whether the infiltration reaches the rectal submucosa. RWC-TVS may be more painful than TVS, therefore it could be used when TVS cannot exclude the presence of rectal infiltration in women with rectovaginal endometriosis [15] (Figure 5).

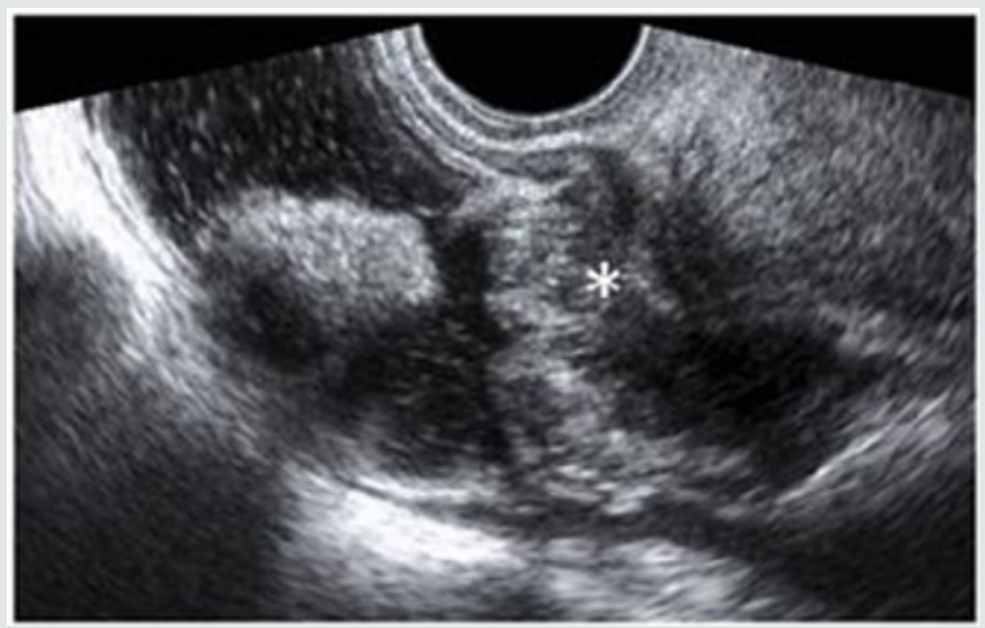

Figure 5: A large rectovaginal nodule infiltrating the bowel muscularis (indicated by the asterisk) demonstrated by Rectal Water Contrast- Transvaginal Sonography (RWC-TVS) [16].

\section{CT \& MSCT}

CT is not the primary imaging modality for evaluation of bowel endometriosis, although it can occasionally demonstrate a stenosing rectosigmoid mass. Multislice CT (MSCT) has a great potential for detecting alterations in the intestinal wall, especially if it is combined with enteroclysis (MSCTe). Biscaldi et al carried out a study on 98 women with symptoms suggestive of colorectal endometriosis and MSCTe identified $94.8 \%$ of bowel endometriotic nodules [1]. Biscaldi et al reported the usefulness of multislice CT combined with distention of the colon by rectal enteroclysis for bowel endometriosis. The sensitivity was $98.7 \%$ and specificity was $100 \%$ in identifying women with intestinal endometriosis. This method is thought to be very helpful for diagnosing intestinal endometriosis, but requires bowel preparation, such as the need for a low-residue diet for $3 \mathrm{~d}$, drinking of 4-6 doses of a granular powder dissolved in $500 \mathrm{~mL}$ of water per dose and intravenous administration of iodinated contrast medium. This technique is thus inappropriate for patients with obstructive symptoms or allergy to iodinated contrast medium [3] (Figures 6 \& 7).
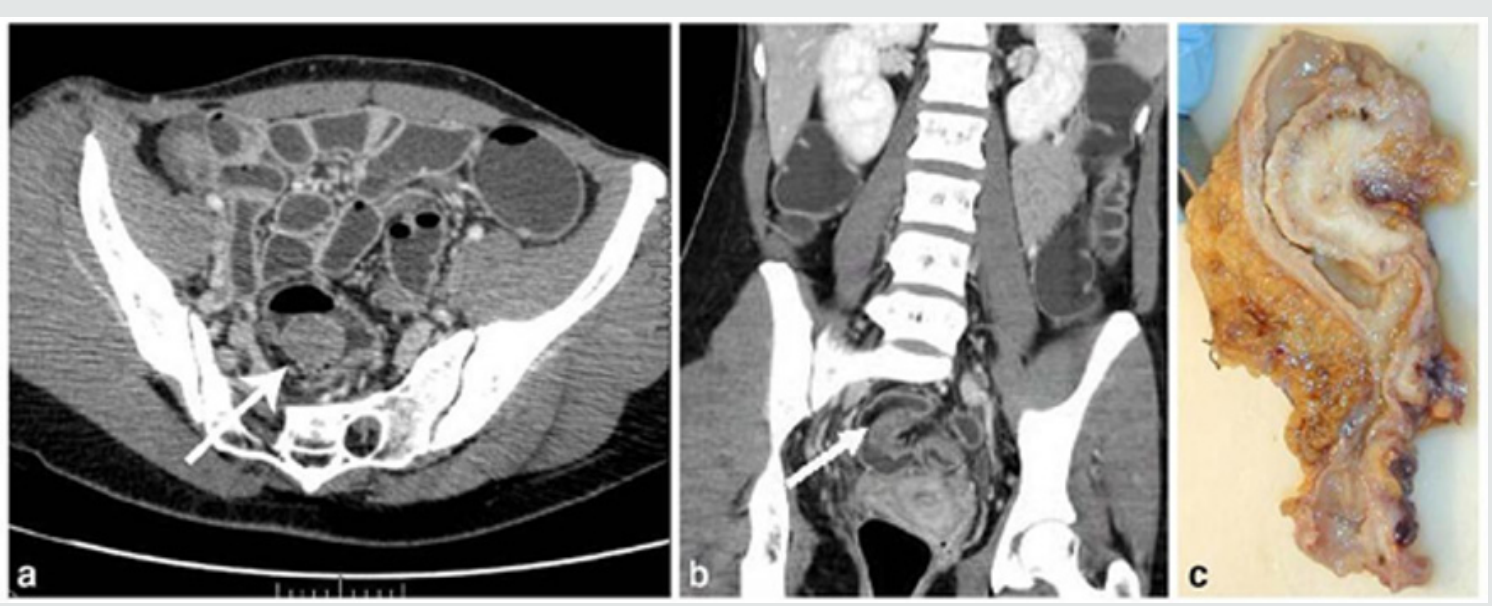

Figure 6: Endometriotic nodule infiltration the muscular layer, A: Axial MSCTe image of the abdomen, the arrow indicates the endometriotic nodule. The lesion is enhanced, and it infiltrates the bowel wall involving the muscular layer. B: Coronal reconstruction demonstrating the extension of the sigmoid endometriotic nodule (indicated by the arrow) C: Formaldehydefixed resected bowel segment, the endometriotic nodule of the sigmoid colon was previously demonstrated by MSCT [14]. 

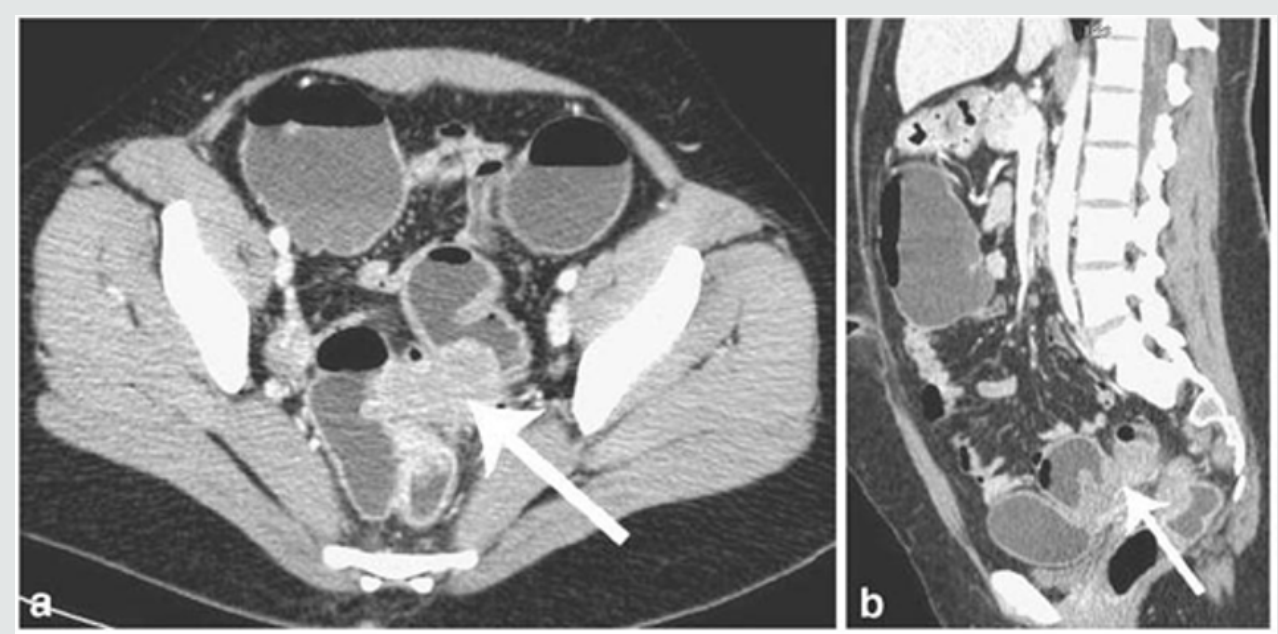

Figure 7: Endometriotic nodule not infiltrating the mucosal layer. A: Axial MSCTe image of the pelvis, the white arrow indicates the nodule. B: Sagittal reconstruction of MSCTe image of the pelvis of the same patient, the white arrow indicates the endometriotic nodule [14].

\section{Magnetic Resonance Imaging (MRI)}

Magnetic resonance imaging (MRI) has a high sensitivity (77\%93\%) in the diagnosis of bowel endometriosis. The depth of rectal wall infiltration by endometriosis is poorly defined by MRI. A combination of MRI and rectal endoscopic ultrasonography (EUS) has recently been proposed. When retroperitoneal infiltration is present, it is mandatory to know if the bowel wall is involved in order to identify patients requiring bowel resection. Both rectal EUS sensitivity and negative predictive value range from $92 \%$ to $100 \%$. The specificity and positive predictive value are rather poor, which are $66 \%$ and $64 \%, 83 \%$ and $94 \%$, respectively, as reported in two different studies [1]. Imaging examination is thus essential for the preoperative diagnosis of intestinal endometriosis, but some reports have described preoperative confusion between this disease and cancer according to colonoscopy and CT with barium enema, particularly in patients with lesions involving the mucosal surface. In such patients, MRI is helpful for differential diagnosis. In a typical endometrial lesion, MRI showed signal hyperintensity on T1-weighted imaging and signal hypointensity on T2-weighted imaging. However, smooth muscle components are reportedly recognized frequently in endometrial lesions. In such lesions, as seen in the present case, MRI indicates signal hypointensity on both T1- and T2-weighted imaging, and differential diagnosis from other diseases such as cancer and gastrointestinal stromal tumor is thus difficult. In fact, Chapron et al reported that MRI specificity for deeply infiltrating endometriosis was $97.9 \%$, but sensitivity was only $76.5 \%$ [3] (Figures 8 \& 9).

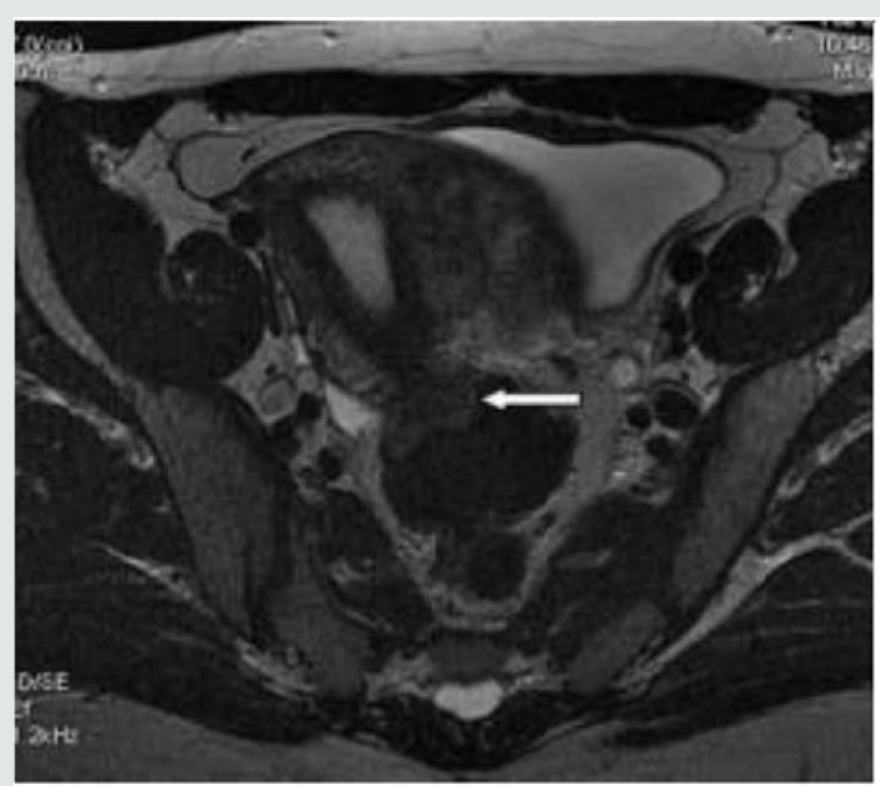

Figure 8: T2- weighted axial view: fecal matter attached to the rectal wall, simulating thickening of the rectal wall [17]. 

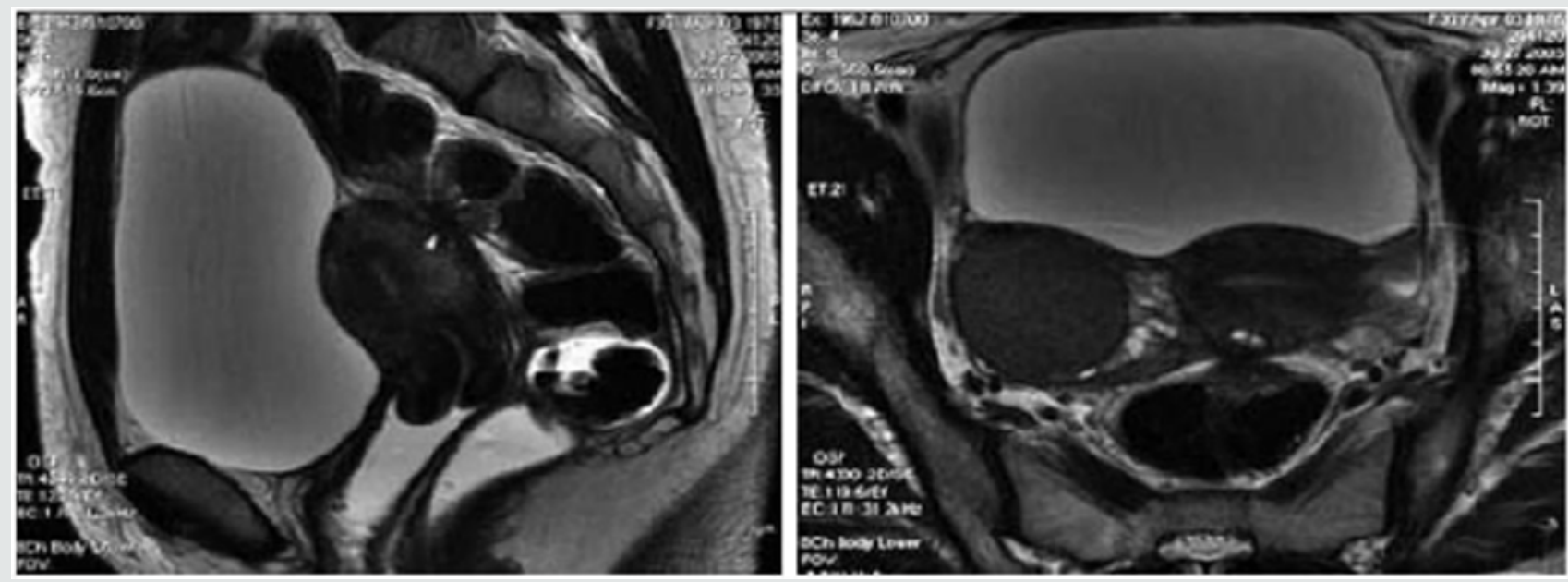

Figure 9: T2- weighted sagittal (a) and axial (b) views.

Nodule of the rectosigmoid junction adhering to the posterior surface of the uterus [17].

\section{Transrectal EUS}

The involvement of the colon is difficult to detect because the implants rarely invade through the intestinal mucosa. For this reason, the rectal ultrasound is of primary importance to assess the rectal involvement [11]. Also, the depth of rectal wall infiltration by endometriosis is poorly defined by MRI. A combination of MRI and rectal endoscopic ultrasonography (EUS) has recently been proposed. When retroperitoneal infiltration is present, it is mandatory to know if the bowel wall is involved in order to identify patients requiring bowel resection [1]. Endoscopic ultrasonography is also a useful and noninvasive examination for the diagnosis of intestinal endometriosis. Sensitivity and specificity are reportedly about $97 \%$ for the diagnosis of rectal involvement in patients with known pelvic endometriosis. In addition, EUS-FNAB provides accurate tissue and may be the only procedure for correct preoperative diagnosis of intestinal endometriosis, but the overall specificity, sensitivity and accuracy of EUS-FNA for neoplasms of the gastrointestinal tract are reportedly 88\%, 89\% and 89\%, respectively [3]. Among these examinations, it is considered that MRI and EUS (and/or EUS-FNAB) are the most useful examinations for intestinal endometriosis. However, it is important to perform valuable examinations for diagnosis of intestinal endometriosis, including radiological, histological and etiological examinations, as the condition basically involves a benign lesion requiring minimally invasive treatment [3] (Figure 10).

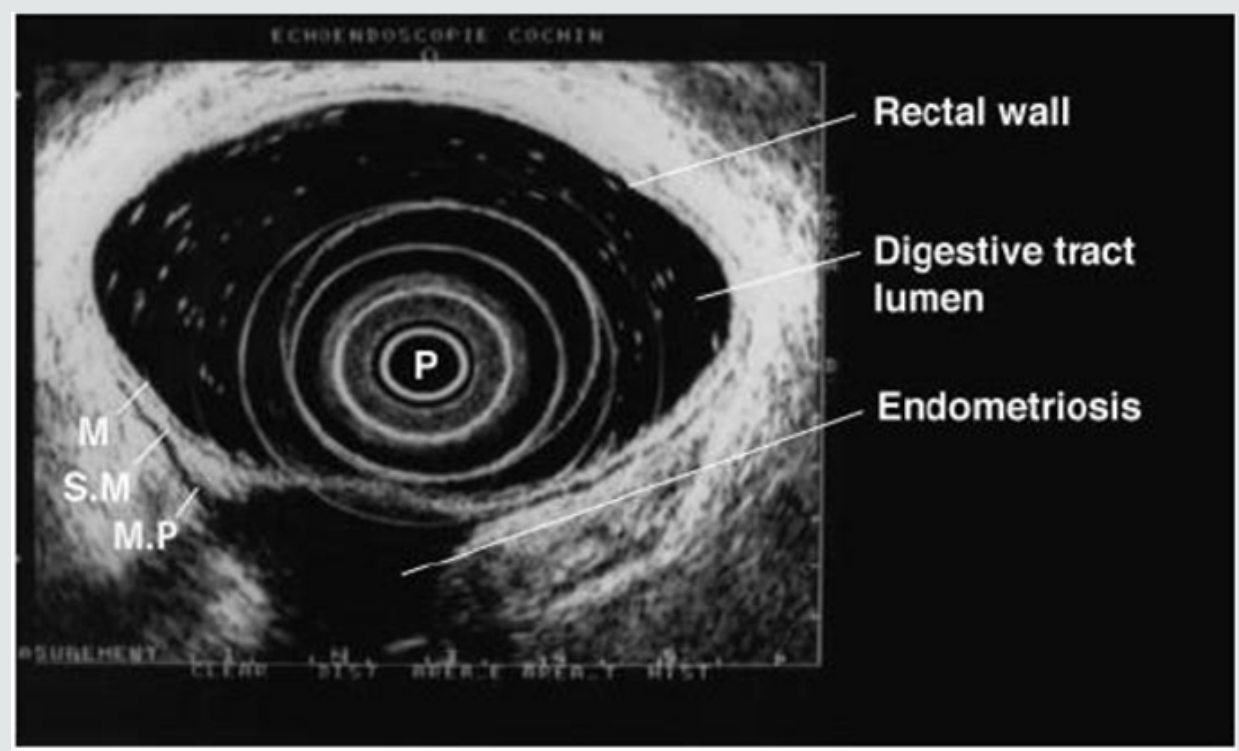

Figure 10: Rectal endoscopic ultrasonography showing a uterosacral endometriosis nodule $(2 \mathrm{~cm} \times 3 \mathrm{~cm})$ with bowel infiltration. $\mathrm{P}=$ probe, $\mathrm{M}=$ mucosa, $\mathrm{SM}=$ submucosa, $\mathrm{MP}=$ muscularis propria [18].

\section{7- Serum Markers}

There is a great interest in the use of serum markers to diagnose endometriosis, but they are not sufficiently accurate for use in clinical practice. Cancer antigen CA-125 has been used to monitor the progress of endometriosis [16]. CA19-9 has a lower sensitivity than CA-125, and cytokine interleukin- 6 may be more sensitive and 
specific than CA-125 [1]. Mol et al reported a systematic review of the diagnosis of endometriosis and concluded that serum CA125 level may be elevated in endometriosis, but this measurement had no value as a diagnostic tool compared to laparoscopy [3].

\section{Laparoscopy}

Laparoscopy is a primary diagnostic and therapeutic tool providing the opportunity to explore the abdominal cavity and obtain biopsies. The magnified vision enables the surgeon to operate with the best possible exposure. Although it was once believed that intestinal endometriosis was best managed by hormonal regimens or surgical castration, the advent of laparoscopic surgery has dramatically changed this approach [11] (Table 2).

Table 2

\section{Summary}

Clinical parameters are insufficient to confirm the diagnosis. Likewise, combining laboratory tests such as CA125 level determinations with imaging methods such as ultrasonography, tomography and magnetic resonance provides relative value for reaching a conclusive diagnosis in the initial stages of endometriosis. Combining laparoscopy with histopathological examinaᄀtion yields greater sensitivity for the definitive diagnosis of the disease and also decreases the diagnostic errors [2].

\section{Treatment}

Despite being a gynecologic pathology, deep infiltrating endometriosis is not of exclusive gynecologic concern. A multidisciplinary approach involving urologists and colorectal surgeons therefore is recommended strongly for complete evaluation and correct management. A minimally invasive approach offers convenient advantages concerning the surgical management of multifocal deep infiltrating endometriosis. Traditionally, radical surgery [17] was considered the best measure to prevent disease relapse. However, because of the prevalence of endometriosis among women of reproductive age and the advances in surgical techniques, minimally invasive conservative surgery now is encouraged more [18]. Treatment must be individualized, taking the clinical problem in its entirety into account, including the impact of the disease and the effect of its treatment on quality of life. Pain symptoms may persist despite seemingly adequate medical and/or surgical treatment of the disease. In such circumstances, a multidisciplinary approach involving a pain clinic and counselling should be considered early in the treatment plan. It is also important to involve the woman in all decisions; to be flexible in diagnostic and therapeutic thinking; to maintain a good relationship with the woman, and to seek advice where appropriate from more experienced colleagues or refer the woman to a centre with the necessary expertise to offer all available treatments in a multidisciplinary context, including advanced laparoscopic surgery and laparotomy [19]. The objective of the treatment in pelvic endometriosis is to cease the endometrial stimulus in order to ameliorate the symptoms. Thus, danozol, gonadotropin- releasing hormones, oral contraceptives, and prostaglandin inhibitors can be used. The conclusive treatment of endometriosis is total abdominal hysterectomy, bilateral salpingo-oophorectomy and removal of all endometrial foci. Because malignant transformation cannot be excluded preoperatively and medical treatment may cause fibrosis, the definitive treatment is surgical. Also, in the case of intestinal obstruction and severe rectal and abdominal pain, surgery is indicated. The main objective of surgery is the resection of the affected bowel segment, enabling the histopathological examination of the resection material. Limited surgery, such as excision or cauterization of superficial lesions, following confirmation through frozen section analysis could be performed. In conclusion, intestinal endometriosis is a disease that may imitate various gastrointestinal system diseases. The definite diagnosis could only be done by histopathologic confirmation, since there are no pathognomonic radiological or colonoscopic findings. In female patients who have unexplained digestive complaints, endometriosis should also be considered in the differential diagnosis [20].

\section{The Treatment of Uncomplicated Intestinal Endometriosis}

It depends on the patient's age and intention to conceive. Bowel resection is indicated if there are symptoms of obstruction or bleeding, and if malignancy cannot be excluded. In patients of childbearing age, resection of the involved colon followed by hormonal treatment may be sufficient; otherwise, hysterectomy and bilateral oophorectomy is the treatment of choice [21]. Medical suppressive therapy may be beneficial in some patients with symptomatic rectovaginal endometriosis, but often it is either ineffective or only temporarily effective, whereas surgical therapy is effective in relieving pain conditions. Other studies have shown that operative therapy of rectovaginal endometriosis does not modify reproductive prognosis but significantly reduces pain and improves quality of life. The best long-term results are obtained after complete excision of the endometriotic tissue [22]. The surgeon's judgment on bowel involvement with the consequence of bowel resection is of the utmost importance [22]. Redwing has suggested a severity scoring system for intestinal endometriosis based on the form of surgical management required: grade I, superficial seromuscular; grade II, partial thickness to mucosa; grade III, full thickness; grade IV, segmental. The surgical approaches to intestinal disease include simple excision (with cautery or laser), mucosal skinning, full thickness disc excision with primary closure, and formal bowel resection [23]. Full thickness disc resection of bowel endometriotic lesion is often incomplete, at least one-third of patients with bowel endometriosis treated by full thickness disc resection have persistent disease. The surgeons must always weigh the risk of potential complications of surgery against the benefit of the complete removal of bowel endometriotic lesions. To date, no clear guideline exists for the pre-operative assessment of patients with suspected endometriosis; therefore, bowel resections should only be performed after a careful pre-operative evaluation of patients' symptoms and a radiological examination of the bowel [23]. Bowel resection can be performed according to previously published criteria (Remorgida et al.): single lesion $>3 \mathrm{~cm}$ in diameter, single lesion infiltrating $>50 \%$ of the bowel wall, three or more lesions infiltrating the muscular layer [15]. 


\section{Operative Technique}

The collaboration of a laparoscopically skilled gynaecologist and colorectal surgeon has been recognised as ideal in the surgical management of colorectal endometriosis. Patients undergo bowel preparation $24 \mathrm{~h}$ prior to surgery with a Fleet ${ }^{\circ}$ ACCU-PREP $®$ Bowel Cleansing System (C.B. Fleet Co., Braeside, Vic., Australia) [24]. Prophylactic anticoagulant therapy was given the evening before the operation, and prophylactic antibiotic therapy was given at the beginning of the operation [25]. Surgery is performed with the patient in the lithotomy position. Five ports are used with placement as shown in Figure 11. Five-millimetre $0^{\circ}$ and 5-mm $30^{\circ}$ endoscopes are used and most dissection is undertaken using the harmonic scalpel (Ethicon Endo-Surgery, Inc., Cincinnati, OH, USA). Pneumoperitoneum is maintained at $12-14 \mathrm{mmHg}$ with a flow rate of $40 \mathrm{~L} / \mathrm{min}$. For an anterior segmental bowel resection, the descending colon is mobilised up to the level of the splenic flexure. The ureter is identified crossing the pelvic brim on the left, and mobilisation continued inferiorly to open the para-rectal space medial to the uterosacral ligament. The right mesocolon is then opened and dissection extended down to the right pararectal space medial to the uterosacral ligament. The right ureter is identified during this dissection. The sigmoid colon is then elevated with bowel grasping forceps, and the space posterior to the sigmoid mesocolon is opened. During this dissection the inferior mesenteric vessels are identified and divided using an endoscopic linear cutter $45 \mathrm{~mm}$ (ATW45 Ethicon Endo-Surgery, Inc.) once the requirement for a bowel resection is established. Further elevation of the sigmoid colon allows the posterior dissection to continue inferiorly to the presacral plane, allowing mobilization of the rectum. Having mobilized the rectosigmoid laterally and posteriorly, the rectum is then dissected free from the posterior cervix. This is the most difficult part of the dissection and an attempt is made to free disease from the posterior cervix and posterior vaginal wall as completely as possible. If there is coexisting invasive uterosacral disease, this is excised end bloc with the affected rectal segment. The inferior dissection is complete when the normal tissue in the rectovaginal septum is encountered. Once the level of rectal transection is identified, the mesorectum is divided at that point leaving the rectal tube. An endoscopic articulating linear cutter $45 \mathrm{~mm}$ (ATG45 Ethicon Endo-Surgery, Inc.) is introduced and applied transversely across unaffected distal rectum. The stapler is fired to separate the affected rectal segment from the distal rectal stump. Two firings may be required. The lower right $12 \mathrm{~mm}$ port site is then converted to a minilaparotomy incision, approximately 3-4 cm in length. The affected rectal segment is then delivered through this wound, clamped and divided above the level of disease. The anvil from an endoscopic curved intraluminal stapler 29 mm (ECS29 Ethicon Endo-Surgery, Inc.) is secured into the proximal colon with a purse-string suture. The proximal segment is then returned to the abdominal cavity and the mini laparotomy wound closed. Pneumoperitoneum is reestablished. The ECS29 is passed transanally, and the distal rectal stump is elevated. The circular stapling device is opened, passing a metal spike through the distal rectal stump adjacent to the staple line. The anvil within the proximal segment is then docked to the spike and the circular stapling device closed. The circular stapling device is then fired to complete the anastomosis. After removing the transanal stapler, an integrity check is performed by distending the rectum with Betadine after occluding the sigmoid colon at the pelvic brim. Further check of integrity is undertaken by instilling air into the rectum after flooding the pelvis with saline. A 17-gauge drain is left in the operative site, after which all ports are removed [24]. Terminal-to-terminal anastomoses were classified according to distance from the anus as high/medium $(>8 \mathrm{~cm})$, low $(5-8 \mathrm{~cm})$ or ultralow $(<5 \mathrm{~cm})$. The choice to perform primary ileostomy or colostomy was based on intraoperative findings [26]. For a disc excision, a lesser degree of descending colon mobilization is required, less posterior rectosigmoid dissection may be required and there is no requirement to divide the inferior mesenteric vessels. The principles of pelvic dissection are otherwise as described. Once the rectal disease has been identified and isolated, a figure of eight suture is placed through the lesion. An ECS33 stapling device is passed transanally with the anvil intact. The device is then opened and angled towards the rectosigmoid lesion. The suture is grasped with laparoscopic forceps, and the disease drawn down into the open stapler. The stapling device is then closed, rotated slightly to ensure that the posterior rectal wall has not become entrapped, and then fired. An anterior arc of rectal wall containing the lesion is therefore removed and the rectal wall stapled in a single action. Rectosigmoid integrity checks are then undertaken as described above [24]. The extent of the lesions as well as the severity of the symptoms justifies the extensive nature of the surgery undertaken. The findings of additional areas of the bowel that are macroscopically normal but microscopically involved, as well as involvement of the lymph nodes, suggest to us that simple local excision of a disc may on occasions be inadequate to remove the whole of the involved area of the bowel [12]. There are no data to justify hormonal treatment prior to surgery to improve the success of surgery [19]. However, according to ESHRE guidelines Postoperative treatment for endometriosis in general might include danazol or a GnRH agonist for 6 months after surgery as it reduces endometriosis associated pain and delays recurrence at 12 and 24 months compared with placebo and expectant management. However, postoperative treatment with a COC is not effective [19].

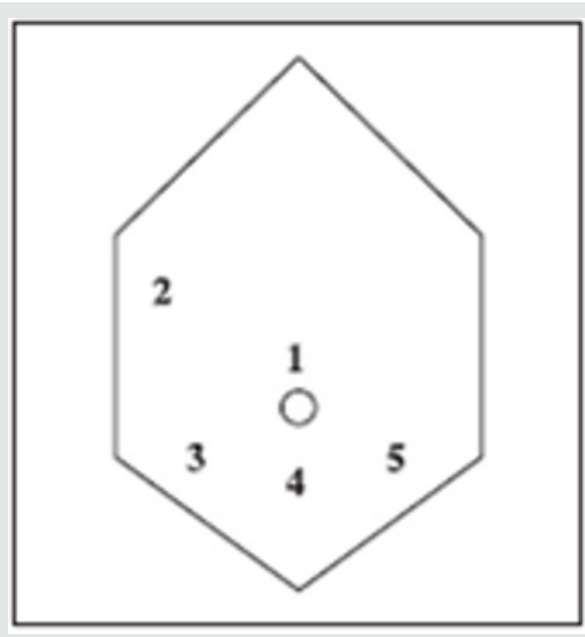

Figure 11: Placement of five port sites used in Surgery. 


\section{Postoperative Complications}

The risk of complications depends on the clinical conditions, such as the level of bowel stenosis, opening of the vaginal wall, the extent of endometriosis infiltration, and the surgeon's experience. Moreover, the possibility of performing this kind of surgery (complete eradication with colorectal surgery) in a referral center reduces the risk of complications and improves clinical outcomes. Indeed, women who undergo intestinal surgery are at higher risk of complications mainly in the short-term, but close surveillance reduces the risk of need for reintervention and allows a good recovery within a few weeks [27]. Complications include:
a) Internal hemorrhage
b) Bowel fistula
c) Vaginal fistula
d) Retention of urine
e) Constipation
f) Abdominal wall hematoma
g) Ureteral injury and stenosis
h) Bladder perforation
i) Uterine perforation
j) Cystitis
k) Adynamic ileus
l) Mechanical bowel obstruction
m) Peritonitis
n) Peritoneal effusion

\section{Outcome after Surgery}

The indications of colorectal resection for endometriosis are controversial, and the likely risk/benefit ratio must be discussed with each patient. No menstrual pelvic pain, pain on bowel movement, cramping, and cyclic rectal bleeding improved or disappeared in all the women concerned, in keeping with previous studies of colorectal resection for endometriosis. dysmenorrhea, dyspareunia, pain on defecation, and no menstrual pelvic pain improved significantly, on the basis of visual analog scores, whereas no impact was noted on pain on bowel movement, lower back pain, or asthenia. Recent results confirm those of Redwing and Wright, showing that women with dysmenorrhea, dyspareunia, pain on defecation, or no menstrual pelvic pain associated with complete endometriotic obliteration of the sac of Douglas are the best candidates for extensive resection [28]. Bowel resection is not completely free of recurrence of endometriosis, but the incidence of recurrence is significantly lower [29]. In conclusion, laparoscopic rectosigmoid resection and end-to-end anastomosis seem safe and effective in women with deep infiltrating colorectal endometriosis, where the bowel lumen is largely restricted, and bowel function is greatly impaired. Results of long-term follow up demonstrate significant reductions in painful and dysfunctional symptoms associated with deep bowel involvement [30]. Laparoscopic segmental colorectal resection for endometriosis is associated with a significant improvement in quality of life and gynecological and digestive symptoms [25].

\section{References}

1. De Ceglie A, Claudio Bilardi, Sabrina Blanchi, Massimo Picasso, Marcello Di Muzio, et al. (2008) Acute small bowel obstruction caused by endometriosis: A case report and review of the literature. World J Gastroenterol 14(21): 3430-3434.

2. De Almeida Filho D, Oliveira LJ, Amaral VF (2018) Accuracy of laparoscopy for assessing patients with endometriosis. Sao Paulo Med J126(6): 305-308.

3. Yoshida M, Watanable Y, Horiuchi A, Yamamoto Y, Sugishita H, et al. (2007) Sigmoid colon endometriosis treated with laparoscopyassisted sigmoidectomy: Significance of preoperative diagnosis. World J Gastroenterol 13(40): 5400-5402.

4. Missmer SA, Hankinson SE, Spiegelman D, Barbieri RL, Malspeis S, et al. (2004) Reproductive history and endometriosis among premenopausal women. Obstet Gynecol 104(5 Pt 1): 965-974.

5. Hediger ML, Hartnett HJ, Louis GM (2005) Association of endometriosis with body size and figure. Fertil Steril 84(5): 1366-1374.

6. Missmer SA, Hankinson SE, Spiegelman D, Barbieri RL, Marshall LM, et al. (2004) Incidence of laparoscopically confirmed endometriosis by demographic, anthropometric, and lifestyle factors. Am J Epidemiol 160: 784-796.

7. Fraser IS (2008) Recognising, understanding and managing Endometriosis. J Hum Reprod Sci 1(2): 56-64.

8. Witz CA (2002) Pathogenesis of Endometriosis. Gynecol Obstet Invest 53(1): 52-62.

9. Anaf V, El Nakadi I, Simon P, Van de Stadt J, Fayt I, et al. (2004) Preferential infiltration of large bowel endometriosis along the nerves of the colon. Human Reproduction 19(4): 996-1002.

10. Marchena GJ, Conde-Martel A, Hemmersbach-Miller M, AlonsoFernandez A (2006) Case report: Metachronic malignant transformation of small bowel and rectal endometriosis in the same patient. World Journal of Surgical Oncology 4: 93.

11. Campagnacci R, Perretta S, Guerrieri M, Paganini AM, De Sanctis A, et al. (2005) Laparoscopic colorectal resection for endometriosis. Surg Endosc 19: 662-664.

12. Abrao MS, Podgaec S, Dias JA, Averbach M, Garry R, et al. (2006) Deeply infiltrating endometriosis affecting the rectum and lymph nodes. Fertility and Sterility 86(3): 543-547.

13. Biscaldi E, Ferrero S, Fulcheri E, Ragni N, Remorgida V, et al. (2007) Multislice CT enteroclysis in the diagnosis of bowel endometriosis. Eur Radiol 17: 211-219.

14. Faccioli N, Manfredi R, Mainardi P, Dalla Chiara E, Spoto E, et al. (2008) Barium Enema Evaluation of Colonic Involvement in Endometriosis. AJR 190: $1050-1054$.

15. Menada MV, V Remorgida, LH Abbamonte, A Nicoletti, N Ragni, et al. (2008) Does transvaginal ultrasonography combined with watercontrast in the rectum aid in the diagnosis of rectovaginal endometriosis infiltrating the bowel? Human Reproduction 23(5): 1069-1075.

16. Jarlot C, Anglade E, Paillocher N, Moreau D, Catala L, et al. (2008) MR imaging features of deep pelvic endometriosis: correlation with laparoscopy. J Radiol 89: 1745-1754.

17. Chapron C, Dumontier I, Dousset B, Fritel X, Tardif D, et al. (1998) Results and role of rectal endoscopic ultrasonography for patients with deep pelvic endometriosis. Human Reproduction 13(8): 2266-2270. 
18. Seracchioli R, Manuzzi L, Mabrouk M, Solfrini S, Frascà C, et al. (2009) A multidisciplinary, minimally invasive approach for complicated deep infiltrating endometriosis: a case report. Fertility and Sterility 93(3): 1007.

19. Kennedy S, Bergqvist A, Chapron C, D Hooghe T, Dunselman G, et al. (2005) ESHRE guideline for the diagnosis and treatment of endometriosis. Human Reproduction 20(10): 2698-2704.

20. Yildirim S, Nursal TZ, Tarim A, Torer N, Bal N, et al. (2005) Colonic obstruction due to rectal endometriosis: Report of a case. Turk J Gastroenterol 16(1): 48-51.

21. Garg NK, Bagul NB, Doughan S, Rowe PH (2009) Case Report: Intestinal endometriosis-A rare cause of colonic perforation. World J Gastroenterol 15(5): 612-614.

22. Zanetti DR, Bartley J, Müller C, Schneider A, Köhler C (2008) Combined vaginal-laparoscopic-abdominal approach for the surgical treatment of rectovaginal endometriosis with bowel resection: a comparison of this new technique with various established approaches by laparoscopy and laparotomy. Surg Endosc 22: 995-1001.

23. Duepree HJ, Senagore AJ, Delaney CP, Marcello PW, Brady KM, et al. (2002) Laparoscopic Resection of Deep Pelvic Endometriosis with Rectosigmoid Involvement. J Am Coll Surg 195: 754-758.

24. WILLS HJ, Reid GD, Cooper MJ, Tsaltas J, Morgan M, et al. (2009) Bowel resection for severe endometriosis: An Australian series of 177 cases. Australian and New Zealand Journal of Obstetrics and Gynaecology 49: 415-418.
25. Dubernard G, Piketty M, Rouzier R, Houry S, Bazot M, et al. (2006) Quality of life after laparoscopic colorectal resection for Endometriosis. Human Reproduction 21(5): 1243-1247.

26. Ruffo G, Scopelliti F, Scioscia M, Ceccaroni M, Mainardi P, et al. (2010) Laparoscopic colorectal resection for deep infiltrating endometriosis: analysis of 436 cases. Surg Endosc 24: 63-67.

27. Minelli L, Ceccaroni M, Ruffo G, Bruni F, Pomini P, et al. (2009) Laparoscopic conservative surgery for stage IV symptomatic endometriosis: short-term surgical complications. Fertility and Sterility 94(4): 1218-1222.

28. Darai E, Thomassin I, Barranger E, Detchev R, Cortez A, et al. (2005) Feasibility and clinical outcome of laparoscopic colorectal resection for endometriosis. American Journal of Obstetrics and Gynecology 192: 394-400.

29. Stepniewska A, Pomini P, Guerriero M, Scioscia M, Ruffo G, et al. (2009) Colorectal endometriosis: benefits of long-term follow-up in patients who underwent laparoscopic surgery. Fertility and Sterility 93(7): 24442446.

30. Seracchioli R, Poggioli G, Pierangeli F, Manuzzi L, Gualerzi B, et al. (2007) Surgical outcome and long-term follow up after laparoscopic rectosigmoid resection in women with deep infiltrating endometriosis. BJOG 114: 889-895.

\section{CC (i) This work is licensed under Creative}

To Submit Your Article Click Here:

Submit Article

DOI: $10.32474 /$ SCSOAJ.2019.03.000155

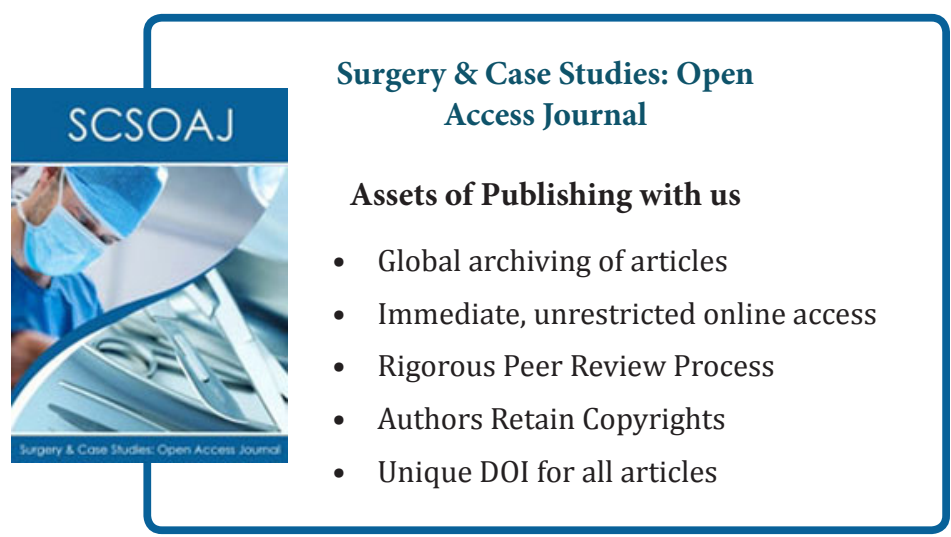

